\title{
Use of the Predictable Quantum Efficient Detector with Light Sources of Uncontrolled State of Polarization
}

Meelis Sildoja, Timo Dönsberg, Henrik Mäntynen, Mikko Merimaa, Farshid Manoocheri, Erkki Ikonen

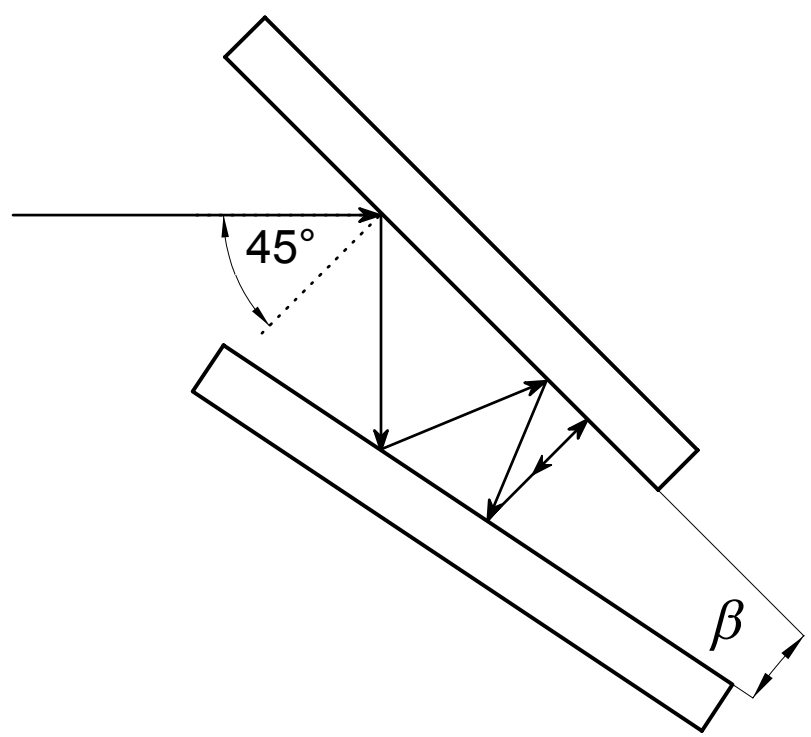





\section{Use of the Predictable Quantum Efficient Detector with Light Sources of Uncontrolled State of Polarization}

Meelis Sildoja, Timo Dönsberg, Henrik Mäntynen, Mikko Merimaa, Farshid Manoocheri, Erkki Ikonen 
Aalto University publication series

SCIENCE + TECHNOLOGY 21/2013

() Meelis Sildoja, Timo Dönsberg, Henrik Mäntynen, Mikko Merimaa, Farshid Manoocheri, Erkki Ikonen

ISBN 978-952-60-5281-6 (pdf)

ISSN-L 1799-4896

ISSN 1799-4896 (printed)

ISSN 1799-490X (pdf)

http://urn.fi/URN:ISBN:978-952-60-5281-6

Unigrafia Oy

Helsinki 2013

Finland

Aalto University and Centre for Metrology and Accreditation 


\section{Author}

Meelis Sildoja, Timo Dönsberg, Henrik Mäntynen, Mikko Merimaa, Farshid Manoocheri, Erkki Ikonen

\section{Name of the publication}

Use of the Predictable Quantum Efficient Detector with Light Sources of Uncontrolled State of Polarization

\begin{tabular}{l} 
Publisher School of Electrical Engineering \\
\hline Unit Department of Signal Processing and Acoustics \\
\hline Series Aalto University publication series SCIENCE + TECHNOLOGY 21/2013
\end{tabular}

Field of research Optical metrology

\section{Abstract}

Analysis of the reflectance losses of the Predictable Quantum Efficient Detector (PQED) operated at room temperature is presented. An efficient method of using the ratio of photocurrents from the two photodiodes of the PQED is developed to determine the reflectance losses without direct measurement of the reflectance for an unknown state of polarization of the incident light. A detailed analysis is presented to estimate the associated reflectance losses for detectors with either seven or nine internal reflections. For the seven-reflection PQED, the relative standard uncertainty component of spectral responsivity due to reflectance loss correction can be reduced typically below $100 \mathrm{ppm}$ with the photocurrent ratio measurement whereas for the nine-reflection PQED the uncertainties remain below $20 \mathrm{ppm}$ in the wavelength range from $400 \mathrm{~nm}$ to $900 \mathrm{~nm}$ with an uncontrolled polarization state of the incident light.

Keywords photodetectors, silicon, optical standards, radiometry, photometry, polarization

\begin{tabular}{lccr}
\hline ISBN (printed) & \multicolumn{4}{c}{ ISBN (pdf) } & $978-952-60-5281-6$ \\
\hline ISSN-L $1799-4896$ & ISSN (printed) $1799-4896$ & ISSN (pdf) & $1799-490 X$ \\
\hline Location of publisher Helsinki & Location of printing Espoo & Year 2013 \\
\hline Pages 20 & urn http://urn.fi/URN:ISBN:978-952-60-5281-6 \\
\hline
\end{tabular}





\section{Contents}

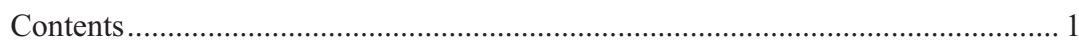

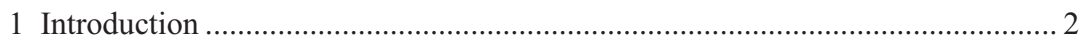

2 PQED with seven internal reflections ......................................................... 3

$2.1 \quad$ Need for improved polarization properties............................................ 3

2.2 Parameter values for the calculations ...................................................... 5

2.3 Reflectance determination by the ratio of photocurrents......................... 6

3 PQED with nine internal reflections.......................................................... 8

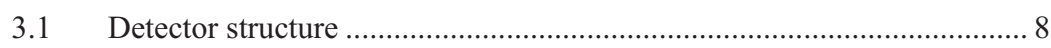

3.2 Reflectance determination by the ratio of photocurrents....................... 10

4 Detailed algorithm for determining the reflectance losses for 7- and 9-reflection PQED.

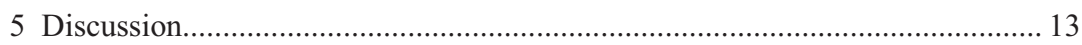

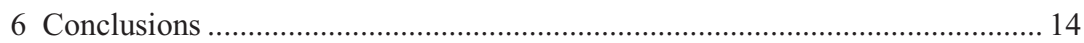

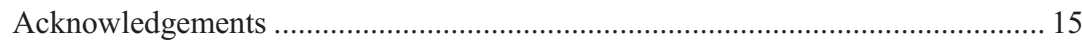

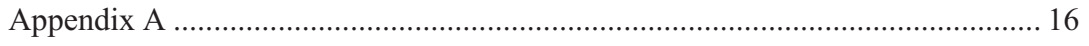

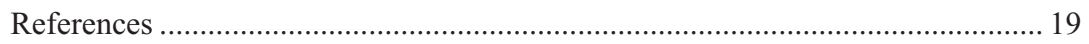




\section{Introduction}

Realization and characterization of the Predictable Quantum Efficient Detector (PQED) with seven internal reflections were recently reported by Sildoja et al [1] and Müller et al [2]. Those results revealed unprecedented accuracies in the determination of the detector spectral responsivity. In suitable conditions the external quantum deficiency (EQD) of the PQED is less than 100 ppm (parts per million). The predicted and measured EQD values agreed within expanded uncertainties, ranging typically between $10 \mathrm{ppm}$ and $180 \mathrm{ppm}$. Some of the limiting factors are related to the reflectance correction of the seven-reflection PQED whose relative responsivity may vary up to $500 \mathrm{ppm}$ depending on the polarization state of the detected light.

The recent work on PQED is based on a series of early publications starting from the important contributions by references [3-5]. In 2003 Geist et al [6] estimated theoretically that a dedicated photodetector based on induced junction silicon photodiodes can register incident photons with uncertainties down to 1 ppm. Detailed analyses of possible detector structures indicated that it should be possible to reduce uncertainties due to reflectance losses of p-polarized laser light to equally low level $[7,8]$. Gran et al [9] carried out thorough modelling of the internal quantum deficiency (IQD) for real induced junction photodiodes using PC1D solar cell software and showed that at low temperatures $(78 \mathrm{~K})$ and at reverse bias mode $(-5 \mathrm{~V})$ the induced junction photodiodes should have IQD smaller than $1 \mathrm{ppm}$ in the wavelength range between $400 \mathrm{~nm}$ to $600 \mathrm{~nm}$. At room temperature the modelled IQD was predicted to be below $100 \mathrm{ppm}$ showing almost constant values at visible wavelengths.

In this paper we first describe the properties of the seven-reflection PQED with light sources of uncontrolled state of polarization, such as monochromator sources operated without polarizing optics. A new diagnostics method to estimate reflectance losses by measuring the photocurrent ratio of the two photodiodes in 
the PQED is introduced. Then we discuss methods to improve the performance of the seven-reflection PQED by introducing the beneficial properties of a ninereflection PQED with emphasis on estimating and reducing the reflectance losses and polarization dependence of the responsivity. The main interest is in PQED operation at room temperature with light sources of uncontrolled state of polarization, although some considerations are presented concerning operation with p-polarized light at low temperatures.

Table 1. Uncertainty components affecting the reflectance and photocurrent ratio values.

The magnitudes of the components are taken from reference [1].

\begin{tabular}{lc}
\hline Standard uncertainty of component $(k=1)$ and its magnitude \\
\hline Angle between photodiodes & $0.4^{\circ}$ \\
Oxide thickness of the $1^{\text {st }}$ diode & $2 \mathrm{~nm}$ \\
Oxide thickness of the $2^{\text {nd }}$ diode & $2 \mathrm{~nm}$ \\
$\mathrm{SiO}_{2}$ index of refraction & $0.2 \%$ \\
$\mathrm{Si}$ index of refraction & $0.5 \%$ \\
$\mathrm{Si}$ extinction coefficient & $5 \%$ \\
Optical thickness of $\mathrm{Si} / \mathrm{SiO}_{2}$ interface layer & $8 \mathrm{~nm}$ \\
\hline
\end{tabular}

\section{PQED with seven internal reflections}

\subsection{Need for improved polarization properties}

The seven-reflection PQE D consists of two custom-made induced junction photodiodes mounted in a wedged light trap configuration with an angle of $15^{\circ}$ between the photodiodes. Thorough characterization of the seven-reflection PQED is given in [1]. Main emphasis therein was the measurement and theoretical modelling of spectral reflectance with p-polarized light, which is defined to be parallel with the plane of incidence of the photodiodes. The use of s-polarized or unpolarized light was not relevant due to the requirement of a Brewster-angled 
entrance window of the PQED for measurements inside a vacuum chamber at low temperatures.

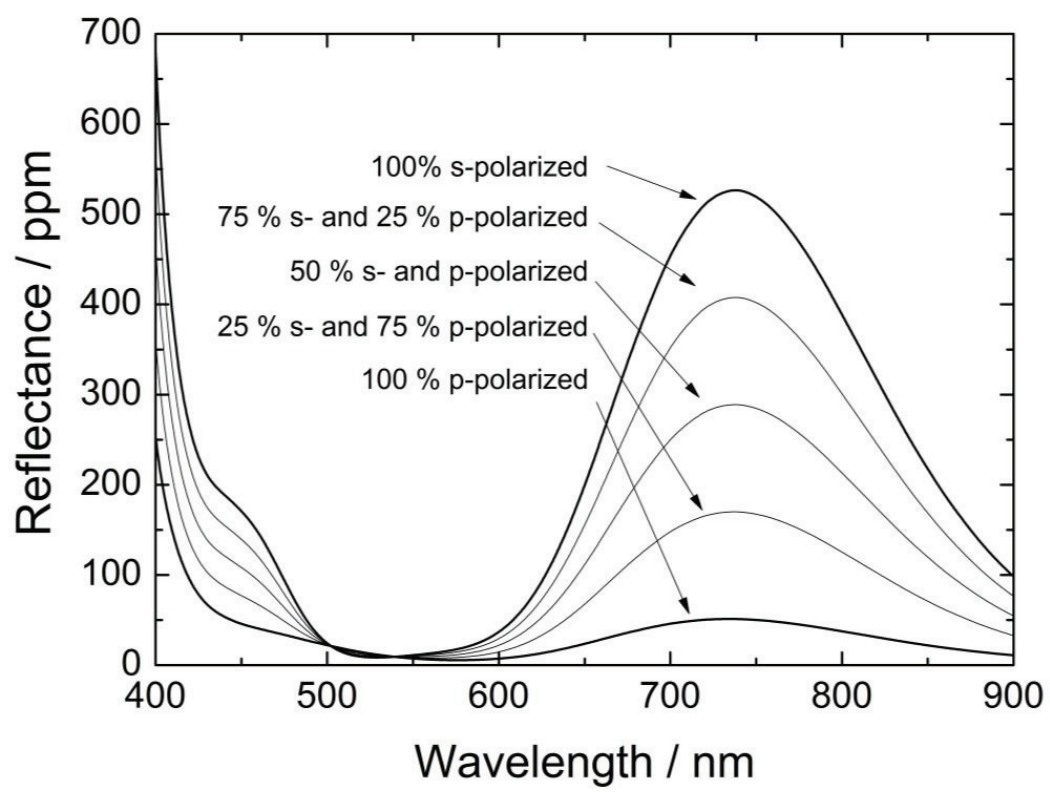

Figure 1. Reflectance of the 7-reflection PQED in the case of incident light with different combinations of s- and p-polarized components (see text for the definition of the polarization components).

The use of PQED with light sources of unknown polarization state becomes of high interest at room temperature, where operation of the PQED without a window is possible using a nitrogen flow system to prevent dust contamination of the photodiodes [10]. The relatively high reflectance of s-polarized light then causes increased uncertainties for light with unknown polarization, especially at short and long wavelengths as shown in Figure 1. To quantitatively describe the effect of the polarization, it is first noted that any polarization state of the incident light has definite normalized s- and p-polarization components, perpendicular and parallel to the plane of incidence, respectively, which are obtained as the squared amplitude of the relative electric field in the corresponding direction. For example, $50 \%$ of 
both s- and p-polarization components in the incident light of Figure 1 can correspond to circularly polarized light, fully unpolarized light, or linearly polarized light with equal s- and p-components.

It is possible to acquire knowledge about the normalized polarization components of light entering into the PQED if one monitors the photocurrents from the two photodiodes separately and calculates the ratio of these values. Then the polarization components at a certain wavelength can be used to obtain an estimate for the reflectance loss of the PQED. This methodology is introduced in the next sub-sections.

\subsection{Parameter values for the calculations}

For calculation of the reflectance and photocurrent ratio, the layered structure of the PQED photodiodes is assumed to be similar to that given in [1]: the substrate is lightly doped (111)-oriented p-type silicon, the oxide thicknesses covering the photodiodes are $301.4 \mathrm{~nm}$ and $220.5 \mathrm{~nm}$, the thickness of the interface layer between $\mathrm{Si}$ and $\mathrm{SiO}_{2}$ is $1 \mathrm{~nm}$, and the thickness of a thin condensed layer of water on top of the $\mathrm{SiO}_{2}$ layer is $1 \mathrm{~nm}$. The refractive indices of silicon, interface layer and water layer are taken from refs. [11-13] whereas the refractive indices for $\mathrm{SiO}_{2}$ are compiled based of refs. $[12,14,15]$. The deviations of the above parameters from those measured and used in [16] are much smaller than the standard uncertainty estimates of the same parameters as listed in [1].

The uncertainty estimation takes into account the largest components affecting the reflectance and photocurrent ratio values. These are the uncertainty of the angle between the photodiodes, the uncertainties of the oxide thicknesses of the photodiodes, the uncertainties of the index of refraction of $\mathrm{Si}$ and $\mathrm{SiO}_{2}$, and the uncertainty of the optical thickness of the interface layer between $\mathrm{Si}$ and $\mathrm{SiO}_{2}$. The magnitudes of these uncertainties are repeated in Table 1 according to reference 
[1]. The alignment of the PQED assumes that the propagating beam inside the detector follows similar paths when entering and leaving the detector.

\subsection{Reflectance determination by the ratio of photocurrents}

Figure 1 indicates that the PQED reflectance depends strongly on the polarization of incident light at the wavelengths between $400 \mathrm{~nm}$ to $490 \mathrm{~nm}$ and between $575 \mathrm{~nm}$ to $900 \mathrm{~nm}$. Calculations show that additionally there is a change in the distribution of the absorbed photons between the two photodiodes as the relative magnitudes of the polarization components change. This distribution can be registered as the photocurrent ratio of the photodiodes. Figure 2 shows the calculated photocurrent ratio for s- and p-polarized light at wavelengths from 400 $\mathrm{nm}$ to $900 \mathrm{~nm}$ where the thin lines indicate the standard uncertainties due to used parameter values. It is seen that for most wavelengths the photocurrent ratio is significantly different for s- and p-polarized light. Only at two regions around 475 $\mathrm{nm}$ to $495 \mathrm{~nm}$ and around $555 \mathrm{~nm}$ to $580 \mathrm{~nm}$ the ratio of the photocurrents is almost equal at any polarization state. At these wavelengths no polarization properties of the incident light can be detected based on photocurrent ratio measurements.

Considering the reflectance associated with the normalized polarization components as shown in Figure 1, the wavelengths from $555 \mathrm{~nm}$ to $580 \mathrm{~nm}$ are conveniently located in the region where the reflectances of both s- and ppolarization components are small staying below $25 \mathrm{ppm}$. More problematic is the wavelength region around $485 \mathrm{~nm}$ where the dependence of reflectance on polarization is most unpredictable. There the reflectance may change from $30 \mathrm{ppm}$ for p-polarized light up to $90 \mathrm{ppm}$ for s-polarized light but the photocurrent ratio reveals no changes. Outside of the discussed wavelength regions the difference in the photocurrent ratio is measurable and the relative polarization components and the associated reflectance are predictable. 


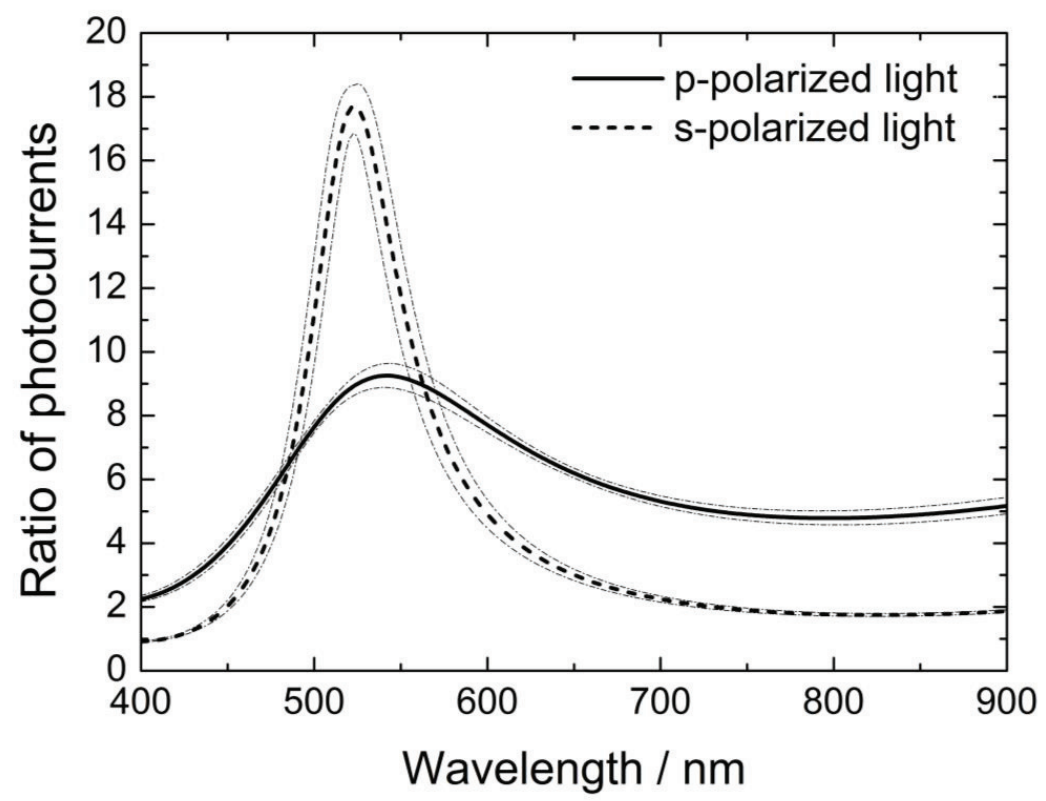

Figure 2. Ratio of photocurrents of the two photodiodes in the 7-reflection PQED if the incident light is polarized in the plane of incidence ( $\mathrm{p}$-polarized) and perpendicular to that (s-polarized). Thin dash-dot lines indicate standard uncertainties due to detector and photodiode parameters (see Table 1 and text for details).

The uncertainty of the reflectance estimated by conducting photocurrent ratio measurements at the wavelengths of $400 \mathrm{~nm}$ and $760 \mathrm{~nm}$ is described in detail in Appendix A. Further calculations show that the reflectance with a standard uncertainty of less than $100 \mathrm{ppm}$ can be determined from the photocurrent ratio measurements at all visible wavelengths above $450 \mathrm{~nm}$. This allows the 7-reflection PQED to be operated at room temperature with light sources of unknown polarization over the wavelength range of $450 \mathrm{~nm}$ to $800 \mathrm{~nm}$ and still maintain almost as low uncertainty of responsivity as previously was available only for p-polarized light sources. However, further reduction of reflectance losses and their uncertainties is required, if direct reflectance measurement is avoided and the uncertainty component due to uncontrolled polarization needs to be made insignificant as compared with other uncertainty components or the high accuracy 
reflectance determination needs to be extended to shorter wavelengths than 450 nm. Methods to meet those requirements are described in next sections.

\section{PQED with nine internal reflections}

\subsection{Detector structure}

The simplest method to reduce the reflectance losses in a trap-like detector is to increase the number of reflections the incident light has to encounter before leaving the detector. Figure 3(a) shows the design of a 9-reflection PQED, where the photodiodes are aligned in such a way that the beam path between the photodiodes is similar for incoming and reflected rays. This can be achieved when the angle between the photodiodes is $11.25^{\circ}$ and the first reflection occurs at $45^{\circ}$ angle of incidence. It is assumed that the physical dimensions of the photodiodes are similar to those used in the 7-reflection PQED [1, 8] i.e. $11 \times 22 \mathrm{~mm}^{2}$ active area. The higher number of 9 reflections is possible by reducing the angle and distance between the photodiodes.

We have analysed the use of the 9-reflection PQED with non-collimated monochromator radiation. Figure 3(b) summarizes the results with a 7-mmdiameter entrance aperture for the beam divergence of $4^{\circ}$ degree cone angle. The upper limiting ray has the ninth reflection at the uppermost corner of the upper photodiode, which determines the minimum distance of $9 \mathrm{~mm}$ between the photodiodes. The lower limiting ray has altogether ten reflections, but the fifth reflection is located close to the lowermost corner of the upper photodiode indicating that the distance between the photodiodes cannot be much larger than 9 $\mathrm{mm}$. In addition, the rays also deviate perpendicular to the plane of incidence with a maximum displacement of $2 \mathrm{~mm}$ for a maximum path length of $60 \mathrm{~mm}$ between the input aperture and the ninth reflection. The transverse photodiode dimension of $11 \mathrm{~mm}$ then just covers the diverging rays perpendicular to the plane of incidence with the aperture diameter of $7 \mathrm{~mm}$. It should be noted that the centre of the 
aperture is displaced $1 \mathrm{~mm}$ down from the mid-point between the uppermost corners of the photodiodes.

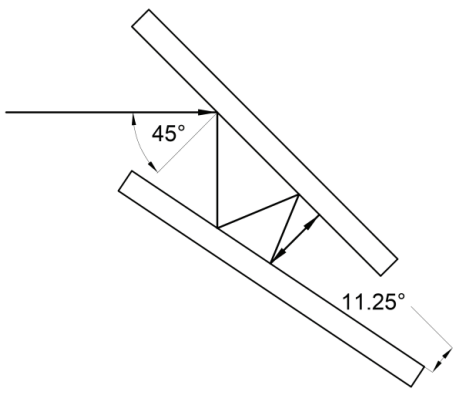

(a)

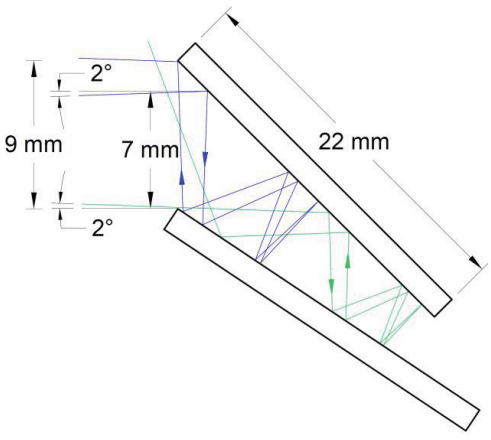

(b)

Figure 3. Assembly of PQED photodiodes in a wedged light trapping configuration having nine reflections before the beam leaves the detector (a) and use of the nine-reflection PQED with a diverging incident beam (b). Coloured (blue and green) rays of incident and reflected light are depicted with the beam divergence of $2^{\circ}$, determining the maximum aperture size of $7 \mathrm{~mm}$ in the vertical direction.

Figure 4 shows the specular reflectance of the 9-reflection PQED with various combinations of s- and p-polarized light in the wavelength range from $400 \mathrm{~nm}$ to $900 \mathrm{~nm}$. For p-polarized light the reflectance is around $1 \mathrm{ppm}$ between the wavelengths of $500 \mathrm{~nm}$ and $640 \mathrm{~nm}$ and it stays below $5 \mathrm{ppm}$ between $430 \mathrm{~nm}$ and $900 \mathrm{~nm}$. The reflectance for s-polarized light is approximately 10 times larger staying below $10 \mathrm{ppm}$ in the wavelength range of $470-640 \mathrm{~nm}$ and reaching the maxima of $70 \mathrm{ppm}$ at $400 \mathrm{~nm}$ and $55 \mathrm{ppm}$ at $730 \mathrm{~nm}$. These low reflectance values provide a good starting point for the reduction of reflectance loss uncertainties when the 9-reflection PQED is used with light sources of uncontrolled polarization. 


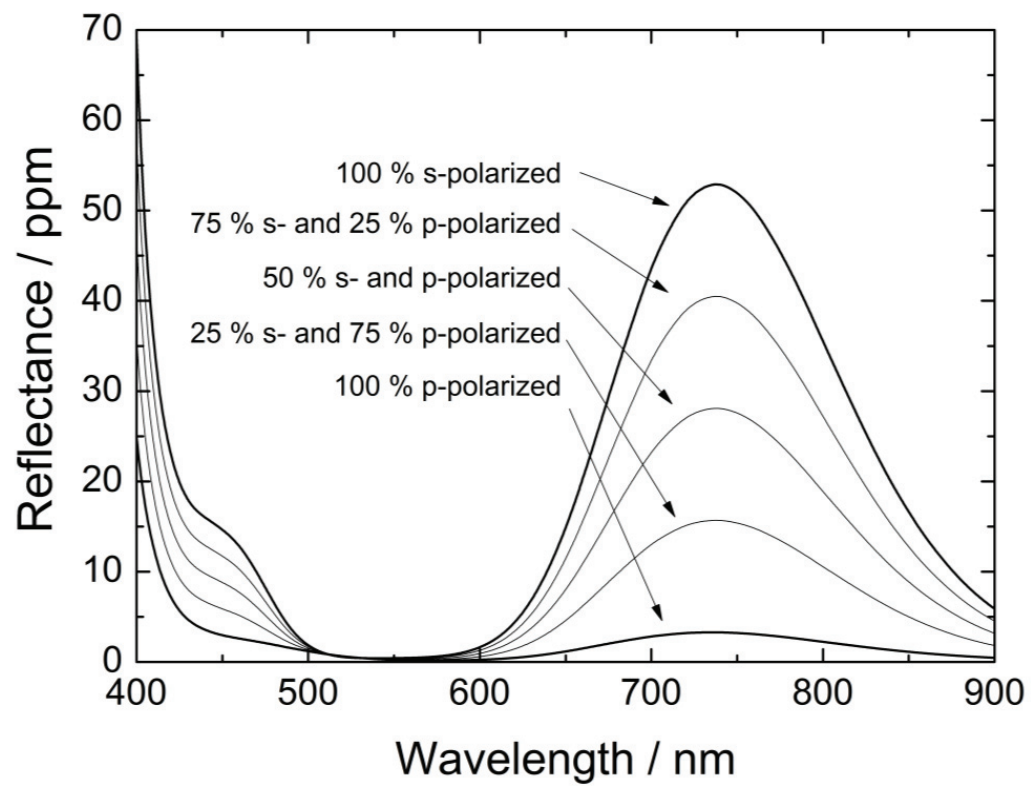

Figure 4. Reflectance of the 9-reflection detector of Figure 3(a) in the case of incident light with different combinations of s- and p-polarized components.

The predicted charge carrier losses of PQED photodiodes at low temperatures are of the order of $1 \mathrm{ppm}$ [9]. The p-polarized light in the 9-reflection trap enables the reflectance losses to be of the similar order of magnitude in a wide range of wavelengths even in the presence of spurious reflectance effects observed in [1] and [2] at low temperatures.

\subsection{Reflectance determination by the ratio of photocurrents}

Polarization dependent spectral reflectances of 9- and 7-reflection traps are similar in shape but differ in magnitude by approximately a factor of ten (Figures 1 and 4). As shown in Appendix A, the largest uncertainty components at the wavelengths of $400 \mathrm{~nm}$ and $760 \mathrm{~nm}$ are reduced by a factor of eight in the determination of reflectance from the photocurrent ratio measurement of the 9- 
reflection PQED as compared with the 7-reflection PQED. Similar improvement is reached at most of the other wavelengths.

In Section 2.3, two wavelength ranges were identified ( $475 \mathrm{~nm}$ to $495 \mathrm{~nm}$ and $555 \mathrm{~nm}$ to $580 \mathrm{~nm}$ ) where the photocurrent ratio measurement does not reveal information about the polarization state of the incident light. This applies also to the 9-reflection PQED due to similarities of photocurrent ratios (see Figures 2 and $5)$.

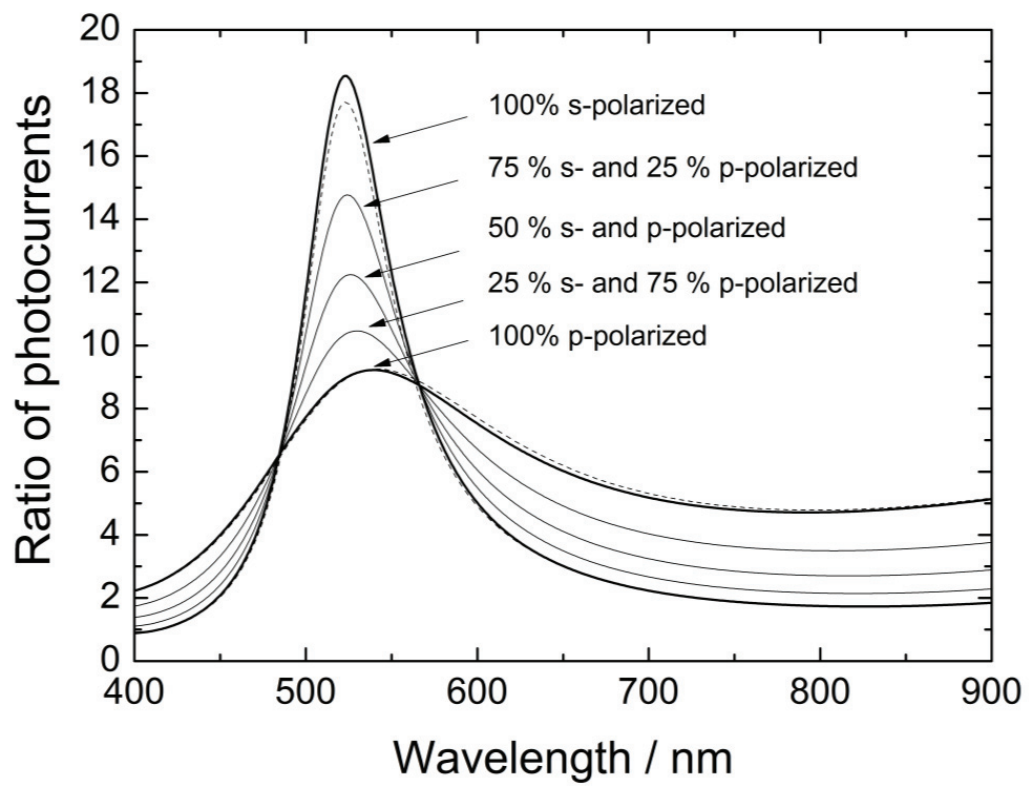

Figure 5. Ratio of photocurrents of the 9-reflection PQED in the case incident light with different combinations of s- and p-polarized components. The relative standard uncertainties of the photocurrent ratios are similar to those presented in Figure 2 for the 7reflection PQED. The thin dashed lines show photocurrent ratios of the 7-reflection PQED from Figure 2 in the case of p- and s-polarized light, indicating that the differences in photocurrent ratios for 7- and 9-reflection PQED are within the standard uncertainties of the predicted photocurrent ratios.

Comparison of Figures 4 and 5 reveals that the reflectances at these wavelength ranges for 9-reflection PQED are relatively small, i.e. below $10 \mathrm{ppm}$, for both 
p- and s-polarized light. This is below the typical uncertainty of reflectance of $\sim 15$ ppm, based on the photocurrent ratio modelling at most of the other wavelengths. These results verify the important conclusion that the reflectance of the 9-reflection PQED can be determined, by photocurrent ratio measurement, for the wavelengths from $400 \mathrm{~nm}$ to $900 \mathrm{~nm}$ within the standard uncertainty smaller than $20 \mathrm{ppm}$, independent of the polarization state on the incident light. The reflectance uncertainty values below $20 \mathrm{ppm}$ are much smaller than the uncertainties due to the predicted charge carrier losses at room temperature.

\section{Detailed algorithm for determining the reflectance losses for 7- and 9-reflection PQED}

Similar algorithms can be used for determining the reflectance losses from the photocurrent ratio of the 7- or 9-reflection PQED. For comparison of the corresponding curve shapes, Figures 1, 2, 4 and 5 show the reflectances and photocurrent ratios of 7- and 9-reflection PQED in various conditions of polarized light. Figures 2 and 5 indicate that photocurrent ratios for 7- and 9-reflection traps are very similar having a maximum difference in the wavelength ranges from 560 $\mathrm{nm}$ to $760 \mathrm{~nm}$ with p-polarized light and from $500 \mathrm{~nm}$ to $570 \mathrm{~nm}$ with s-polarized light. The latter region is insignificant for reflectance estimation since it resides in the wavelength range where the PQED reflectance is in any case low for both sand p-polarization. In the former region the difference is within the uncertainty of the photocurrent ratio modelling.

Any polarization state of the incident light can be decomposed to the components parallel and perpendicular to the plane of incidence. From a plot like Figure 5, it is possible to estimate the composition of s- and p-polarized components in the incident light if the wavelength and PQED photocurrent ratio are known. The corresponding reflectances can be found from Figures 1 and 4 using the estimated composition of polarization components. For example, if the 
measured photocurrent ratio at $700 \mathrm{~nm}$ is 2.50 , such ratio value can be located in the middle of the two lowest lines in Figure 5. Then the associated reflectance can be read using the corresponding lines in Figure 4. For the 9-reflection PQED the reflectance value is in the middle of approximate values of $35 \mathrm{ppm}$ and $45 \mathrm{ppm}$ at about $40 \mathrm{ppm}$. Neglecting the uncertainty in the measured photocurrent ratio, the standard uncertainty of this estimate is $3 \mathrm{ppm}$, as determined by the standard uncertainty of the location of the curves in Figures 4 and 5.

\section{Discussion}

Estimating the reflectance of PQED by measuring individual photocurrents from the two photodiodes avoids the need for developing an experimental arrangement for direct reflectance correction measurement. This is of high interest in spectral power measurements when using quasi-monochromatic light sources with unknown polarization, such as monochromator-based setups where multiple mirrors are used to collimate and align the light beam. Also some laser sources do not have their polarization plane fully vertical or horizontal or the radiated light is randomly or elliptically polarized. The photocurrent ratio measurement and/or use of the 9-reflection PQED greatly help in measurements with light sources of uncontrolled polarization state. Furthermore, the 9-reflection PQED allows use of a 7-mm-diameter entrance aperture with a beam divergence cone angle close to $4^{\circ}$.

A relevant question is whether there is any improvement to be expected with even larger number of reflections in the PQED. An 11-reflection design can be implemented to discard any concerns about the on-axis reflectance losses [8]. This, on the other hand, sets strict limitations to the size of the entrance aperture when using similar sized photodiodes as in the present PQED. We have estimated that the maximum distance of the photodiodes at the entrance plane can be $8 \mathrm{~mm}$ for the 11-reflections and well collimated incident beam. An entrance aperture of this size requires a precisely adjusted angle between the photodiodes and perfect 
alignment of the trap. Minor variations in angular tolerances or tilts of the detector from the ideal alignment may cause the multiply reflected beam either to escape via the back end of the trap structure or produce undesired travel paths for the laser beam. A positive finding is that the high number of 11 reflections allows using large photodiodes with almost any oxide thickness between $100 \mathrm{~nm}$ and $400 \mathrm{~nm}$ to maintain low reflectance.

\section{Conclusions}

We have investigated the reflectance losses and reduction of their uncertainty without direct reflectance measurement of the Predictable Quantum Efficient Detector (PQED), used with light sources of uncontrolled state of polarization. By the photocurrent ratio measurement it is possible to estimate the relative s- and ppolarization components in the incident light. The knowledge of these components allows us to calculate the reflectance of the wedged-trap structure of the PQED. The uncertainty of the reflectance is affected by the uncertainty of the photocurrent ratio measurement, the material parameters of the PQED photodiodes and the uncertainty of the angle between the photodiodes. The analysis was carried out for the 7-reflection and 9-reflection PQED in the wavelength range from $400 \mathrm{~nm}$ to $900 \mathrm{~nm}$. Calculations show that our method allows estimating the reflectance with an uncertainty below $100 \mathrm{ppm}$ for the 7-reflection PQED in the wavelength range from $450 \mathrm{~nm}$ to $900 \mathrm{~nm}$ and below $20 \mathrm{ppm}$ for the 9-reflection PQED in the wavelength range from $400 \mathrm{~nm}$ to $900 \mathrm{~nm}$. Additionally the design of the 9-reflection PQED reduces the absolute reflectance by about a factor of ten as compared with the reflectance of the 7-reflection PQED. These achievements allow the wedged-trap design of the PQED to be used with monochromator based and other light sources where the polarization properties of the beam are unknown and

still maintain the low uncertainty previously available only for fully s- or ppolarized light. 


\section{Acknowledgements}

The authors acknowledge support by the Academy of Finland grant no 126773. The research leading to these results has received funding from the European Community's Seventh Framework Programme, ERA-NET Plus, under Grant Agreement No 217257. 


\section{Appendix A}

This Appendix describes determination of the reflectance loss from the photocurrent ratio for the 7- and 9-reflection PQED at the wavelengths of $400 \mathrm{~nm}$ and $760 \mathrm{~nm}$. These wavelengths are selected because of the high values of spolarized reflectance.

Figure 6(a) shows the calculated photocurrent ratio for the 7-reflection PQED as a function of the normalized s-polarized component. The reflectances on the right-hand-side vertical axis can be estimated using the photocurrent ratio which determines the polarization component on the horizontal axis. At the wavelength of $760 \mathrm{~nm}$ the photocurrent ratio can vary from 4.85(20) with p-polarized light down to $1.86(6)$ with s-polarized light, where the numbers in parenthesis denote the standard uncertainty in the last digits due to the uncertainty corridors of Figure 6. The normalized s-polarized component on the horizontal axis for such photocurrent ratios would be in the ranges of $0 \%$ to $7 \%$ and of $92 \%$ to $100 \%$, respectively. The reflectances with these polarization components vary from $40 \mathrm{ppm}$ to $85 \mathrm{ppm}$ and from $425 \mathrm{ppm}$ to $550 \mathrm{ppm}$, respectively. At the wavelength of $400 \mathrm{~nm}$ the variation of the photocurrent ratio can be from 2.25(10) to $0.92(3)$ with p- and s-polarized light, respectively. In the same way as for the $760 \mathrm{~nm}$ wavelength, the corresponding reflectances can then be estimated to vary from $200 \mathrm{ppm}$ to $325 \mathrm{ppm}$ and from $530 \mathrm{ppm}$ to $790 \mathrm{ppm}$, respectively. Thus the maximum variation in reflectance is $\pm 130 \mathrm{ppm}$ at the wavelength of $400 \mathrm{~nm}$, corresponding to the standard uncertainties in the photocurrent ratio modelling. This occurs close to spolarized light.

Figure 6(b) shows the ratio of photocurrents and corresponding reflectances of the 9-reflection PQED as a function of the normalized s-polarized component. At the wavelength of $760 \mathrm{~nm}$, the photocurrent ratio can be as large as $4.75(25)$ with p-polarized light and as low as $1.85(10)$ with s-polarized light. In the same way as for the 7-reflection PQED, the corresponding reflectances can then be estimated to 
vary from $2.5 \mathrm{ppm}$ to $7.5 \mathrm{ppm}$ and from $40 \mathrm{ppm}$ to $56 \mathrm{ppm}$, respectively. At the wavelength of $400 \mathrm{~nm}$, the variation of the photocurrent ratio is from 2.23(10) with p-polarized light to $0.89(5)$ with s-polarized light, corresponding to reflectances from $16 \mathrm{ppm}$ to $34 \mathrm{ppm}$ and from $50 \mathrm{ppm}$ to $83 \mathrm{ppm}$, respectively. The maximum change in reflectance due to the uncertainty of photocurrent ratio modelling in Figure $6(\mathrm{~b})$ is $33 \mathrm{ppm}$ at the wavelength of $400 \mathrm{~nm}$, a factor of eight smaller than for the 7-reflection PQED. 


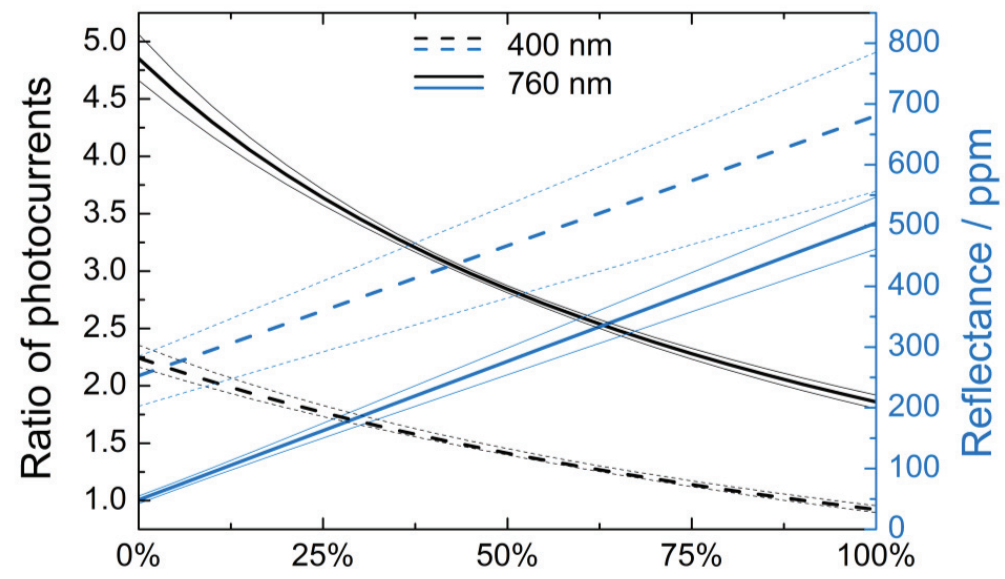

(a) Normalized s-polarized component

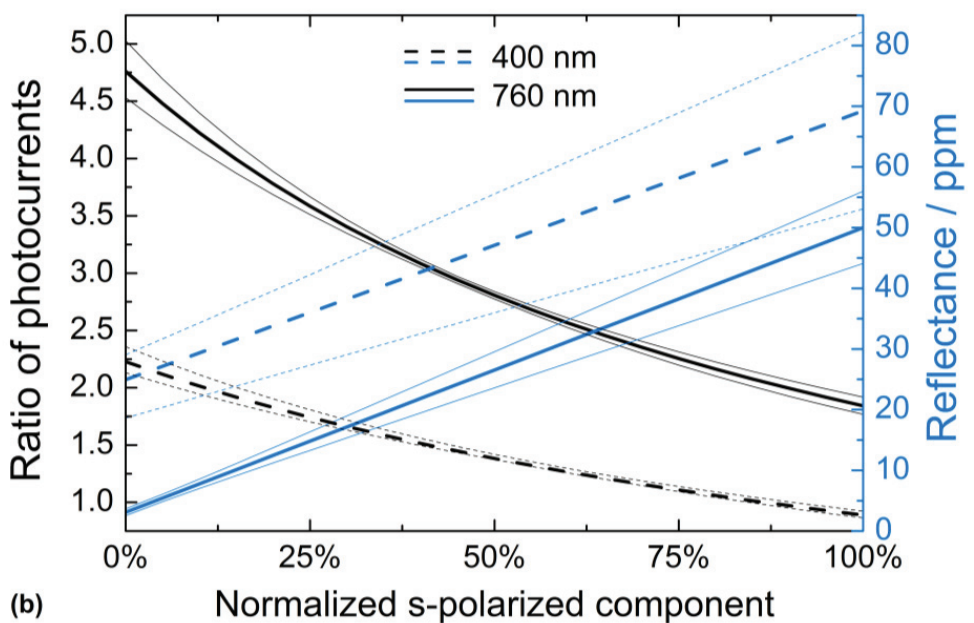

Figure 6. Ratio of photocurrents and corresponding reflectances of the 7-reflection PQED (a) and of the 9-reflection PQED (b) as a function of polarization. Thinner lines indicate the standard uncertainty corridors due to detector and photodiode parameters of Table 1. 


\section{References}

[1] Sildoja M, Manoocheri F, Merimaa M, Ikonen E, Müller I, Werner L, Gran J, Kübarsepp T, Smid M and Rastello M L 2013 Predictable quantum efficient detector: I. Photodiodes and predicted responsivity Metrologia 50 385-394

[2] Müller I, Johannsen U, Linke U, Socaciu-Siebert L, Smid M, Porrovecchio G, Sildoja M, Manoocheri F, Ikonen E, Gran J, Kübarsepp T, Brida G and Werner L 2013 Predictable quantum efficient detector: II. Characterization and confirmed responsivity Metrologia 50 395-401

[3] Hansen T 1978 Silicon UV-photodiodes using natural inversion layers Physica Scripta 18 471-475

[4] Geist J, Liang E and Schaefer A R 1981 Complete collection of minority carriers from the inversion layer in induced junction diodes Journal of Applied Physics 52 4879-4881

[5] Zalewski E F and Duda C R 1983 Silicon photodiode device with 100\% external quantum efficiency Applied optics 22 2867-2873

[6] Geist J, Brida G and Rastello M L 2003 Prospects for improving the accuracy of silicon photodiode self-calibration with custom cryogenic photodiodes Metrologia 40 S132-S135

[7] Sildoja M, Manoocheri F and Ikonen E 2008 Reducing photodiode reflectance by Brewster-angle operation Metrologia 45 11-15

[8] Sildoja M, Manoocheri F and Ikonen E 2009 Reflectance calculations for a predictable quantum efficient detector Metrologia 46 S151-S154

[9] Gran J, Kübarsepp T, Sildoja M, Manoocheri F, Ikonen E and Müller I 2012 Simulations of a predictable quantum efficient detector with PC1D Metrologia 49 S130-S134

[10] Dönsberg T, Sildoja M, Manoocheri F, Merimaa M, Petroff L and Ikonen E Primary standard of optical power based on induced-junction silicon photodiodes for operation at room temperature (in preparation)

[11] Green M A 2008 Self-consistent optical parameters of intrinsic silicon at $300 \mathrm{~K}$ including temperature coefficients Solar Energy Materials and Solar Cells 92 1305-1310

[12] Herzinger C, Johs B, McGahan W, Woollam J A and Paulson W 1998 Ellipsometric determination of optical constants for silicon and thermally grown silicon dioxide via a multi-sample, multi-wavelength, multi-angle investigation Journal of Applied Physics 83 3323-3336

[13] Asay D B and Kim S H 2005 Evolution of the adsorbed water layer structure on silicon oxide at room temperature The journal of physical chemistry. B 109 16760-3 
[14] Aspnes D and Theeten J 1979 Optical properties of the interface between Si and its thermally grown oxide Physical Review Letters 43 1046-1050

[15] Taft E A 1978 The optical constants of silicon and dry oxygen oxides of silicon at 5461A Journal of The Electrochemical Society 125 968-971

[16] White M, Lolli L, Brida G, Gran J and Rajteri M 2013 Optical constants and spatial uniformity of thermally grown oxide layer of custom, inducedjunction, silicon photodiodes for a predictable quantum efficient detector Journal of Applied Physics 113243509 

ISBN 978-952-60-5281-6 (pdf)

ISSN-L 1799-4896

ISSN 1799-4896

ISSN 1799-490X (pdf)

Aalto University

School Electrical Engineering

Department of Signal Processing and Acoustics www.aalto.fi
BUSINESS +

ECONOMY

ART +

DESIGN +

ARCHITECTURE

SCIENCE +

TECHNOLOGY

CROSSOVER

DOCTORAL

DISSERTATIONS 